\title{
CURCUMA LONGA L., O AÇAFRÃO DA TERRA, E SEUS BENEFÍCIOS MEDICINAIS
}

\author{
Juliana Pelissari Marchi ${ }^{1,3}$ \\ Luana Tedesco ${ }^{2,3}$ \\ Ailton da Cruz Melo ${ }^{4}$ \\ Andressa Caroline Frasson ${ }^{5}$ \\ Vivian Francielle França ${ }^{6}$ \\ Samantha Wietzikoski Sato ${ }^{7}$ \\ Evellyn Claudia Wietzikoski Lovato ${ }^{8}$
}

MARCHI, J. P.; TEDESCO, L.; MELO, A. da C.; FRASSON, A. C.; FRANÇA, V. F.; SATO, S. W.; LOVATO, E. C. W. Curcuma longa L., o açafrão da terra, e seus benefícios medicinais. Arq. Cienc. Saúde UNIPAR, Umuarama, v. 20, n. 3, p, 189-194, set./dez. 2016.

\begin{abstract}
RESUMO: Plantas medicinais são de grande importância nos estudos para elaboração de fármacos de origem natural. A Curcuma Longa Linn é uma planta pertencente a espécie Zingiberaceae, cujo nome científico é Curcuma longa L. É popularmente conhecida como açafrão, açafrão da terra, gengibre amarela e raiz de sol. Possui aspecto rizomatoso e forte tonalidade amarela. Originária da ásia e cultivada em regiões tropicais e subtropicais, é muito utilizada na culinária e medicina popular. Esta revisão bibliográfica teve por objetivo descrever os principais usos medicinais da Curcuma longa L. É utilizada como tempero, especialmente na região continental da Índia. Sendo consumido principalmente na forma de pó seco, utilizado para coloração alimentícia devido à sua forte coloração amarela, associada aos efeitos terapêuticos e dietéticos Os pigmentos responsáveis por essa coloração forte, pertencem à classe dos diferoluilmetano, representados pela curcumina, se tornando bastante utilizada como corante. Os rizomas da cúrcuma são processados e deles se extraem óleo essencial, curcumina e fécula. Possui diversas atividades farmacológicas, destacando seus principais potenciais terapêuticos, tais como; atividades anti-inflamatória, antiviral, antibactericida, antioxidante, antifúngica, anticarcinogênica, entre outras ações. Estudos indicaram que a curcumina apresenta efeitos neuroprotetores no tratamento da Doença de Alzheimer e de Parkinson, prevenindo a inflamação e o dano oxidativo. A pesquisa permitiu verificar a importância da Curcuma longa L. e seu grande potencial de utilização, contendo, principalmente, efeito anti-inflamatório e provável efeito neuroprotetor com potencial para o desenvolvimento de medicamentos. Diante disso, fazem-se necessários estudos, para avaliar seu potencial como planta medicinal.
\end{abstract}

PALAVRAS-CHAVE: Ação anti-inflamatória. Ação antioxidante. Benefícios medicinais. Curcuma longa L. Curcumina.

\section{CURCUMA LONGA L., EARTH SAFFRON AND ITS MEDICAL BENEFITS}

\begin{abstract}
Medicinal plants are of great importance in studies for the preparation of naturally occurring drugs. Curcuma Longa Linn is a plant belonging to the Zingiberaceae species, whose scientific name is Curcuma longa L.. It is popularly known as saffron, earth saffron, yellow ginger and sun root, with a rhizomatous aspect and strong yellow hue. It is originally from Asia, cultivated in tropical and subtropical regions, and widely used in cooking and folk medicine. This literature review aimed to describe the main medicinal uses of $C u r c u m a$ Longa L. It is used as a spice, especially in mainland India. Being mainly consumed as a dry powder, used for food coloring due to its strong yellow color associated with therapeutic and dietary effects. Its pigments are responsible for this strong color, belong to the diferuloylmethane class represented by curcumin, becoming widely used as food coloring. The turmeric rhizomes are processed and essential oil, curcumin and starch are extracted from it. It presents several pharmacological activities, such as anti-inflammatory, antiviral, antibacterial, antioxidant, antifungal and anticarcinogenic activities, among others. Studies have shown that curcumin has neuroprotective effects in the treatment of Alzheimer's disease and Parkinson's disease, preventing inflammation and oxidative damage. Research has shown the importance of Curcuma Longa L. and its great use potential. It presents anti-inflammatory and possible neuroprotective effects with potential for drug development. Therefore, further studies are necessary in order to assess its potential as a medicinal plant.
\end{abstract}

KEYWORDS: Anti-inflammatory action. Antioxidant action. Curcuma longa L., Curcumin. Medical benefits.

\section{Introdução}

A aplicação de planta com finalidade medicinal é antiga. Inicialmente seu uso ocorria junto a rituais e orações, sendo, considerado o único meio tradicional de prevenção e/ou tratamento de patologias e lesões dentro de comunidades tradicionais (FIRMO et al., 2011; CARVALHOS, 2012). Este conhecimento foi repassado de geração em geração. A partir de então, por meio da etnobotânica foram descobertos alguns fitoterápicos e medicamentos fitoterápicos empregados na medicina (FIRMO et al., 2011; GIRALDI; HANAZAKI, 2010).

A fitoterapia surgiu na China por volta de 3000 a.C., onde foram descritas as propriedades da cânfora e do ginseng e desenvolvidos laboratórios para pesquisas científicas (TORQUATTO, 2013). Sua definição refere-se ao estudo de plantas medicinais e suas aplicações na prevenção, na atenuação ou no tratamento de patologias, dentro de um contexto

DOI: https://doi.org/10.25110/arqsaude.v20i3.2016.5871

${ }^{1}$ Mestranda do Programa de Pós-graduação em Plantas Medicinais e Fitoterápicas na Atenção Básica da Universidade Paranaense - Umuarama.

${ }^{2}$ Mestranda do Programa de Pós-graduação em Biotecnologia Aplicada a Agricultura da Universidade Paranaense - UNIPAR - Umuarama.

${ }^{3}$ Professora Assistente da Universidade Paranaense - UNIPAR - Francisco Beltrão.

${ }^{4}$ Acadêmico do Curso de Psicologia, PEBIC/ CNPq/ UNIPAR.

${ }^{5}$ Acadêmica do Curso de Psicologia, PIBIC/ UNIPAR.

${ }^{6}$ Professora Adjunta da Universidade Paranaense - UNIPAR; Doutora em Nutrição UFSC.

${ }^{7}$ Professora Adjunta da Universidade Paranaense - UNIPAR; Mestre em Farmacologia UFPR

${ }^{8}$ Docente do Programa de Pós-graduação em Plantas Medicinais e Fitoterápicas na Atenção Básica da Universidade Paranaense - UNIPAR; Doutora em Farmacologia UFPR.

Endereço para correspondência: Pç. Mascarenhas de Moraes, 4282 - Zona III. CEP: 87.502-210. Fone: (44) 3621-2828. E-mail: evellyn@unipar.br 
holístico (TORQUATTO, 2013; ROSSATO et al., 2012).

$\mathrm{O}$ desenvolvimento de fitoterápicos inclui o extrato de determinada planta, com todos os compostos bioativos ali presentes, porém, de uma forma padronizada, com dosagem, riscos e benefícios estabelecidos (TORQUATTO, 2013; ELDIN; DUNFORD, 2001). Enquanto que, o desenvolvimento de medicamentos fitoterápicos usa o conhecimento etnobotânico, etnomédico aliados à tecnologia farmacêutica, a fim de obter controle de qualidade, identificação, quantificação e isolamento de compostos bioativos, que permitem a fabricação de fitomedicamentos (CARVALHOS, 2012; ELDIN; DUNFORD, 2001). Para ambas as situações a base do desenvolvimento do produto é a planta. Portanto, a área de plantas medicinais e fitoterapia vêm se desenvolvendo e deve estar fundamentada a partir de conhecimentos científicos que envolvem fisiologia, fisiopatologia, farmacologia, química orgânica e bioquímica (ABRANCHES, 2015; TORQUATTO, 2013).

Neste contexto, verifica-se que a área de plantas medicinais e fitoterapia vêm ganhando notoriedade. Para isto, o Brasil apresenta a Relação Nacional de Plantas Medicinais de Interesse ao Sistema Único de Saúde (RENISUS). Nesta relação destaca-se a Curcuma longa L., esta por sua vez, apresenta conhecimento etnobotânico e uso etnofarmacológico amplo e conta com diversos estudos nacionais e internacionais (MUNIZ et al., 2012).

A Curcuma longa L. é usada há anos. Seu uso na Índia é datado cerca de 4000 anos a.C., na China foi mencionada no século VII, nos países Árabes no século X e introduzida na Europa no século XIII. Inicialmente era apreciada por seu valor alimentício com propriedades similares ao gengibre, posteriormente ganhou espaço por suas propriedades etnomedicinais que incluem: atividade hepática, gastroprotetora, anti-inflamatória, antimicrobiana, anti-HIV, hipolipemiante, hipoglicemiante, antiagregante, dermatológica, oftalmológica, antioxidante, em oncologia, no sistema respiratório, no sistema reprodutor, no sistema digestório e no sistema nervoso central (ALONSO, 2016). Desta forma, verifica-se que a Curcuma longa L. apresenta diversas ações, portanto, é uma planta que deve continuar sendo estudada, para elucidar possíveis lacunas e sanar dúvidas. Assim, esta revisão bibliográfica tem por objetivo descrever os principais usos medicinais da Curcuma longa $L$.

\section{Desenvolvimento}

\section{Classificação Botânica}

A Curcuma longa L. é uma planta pertencente à família das Zingiberaceae (GRANDI, 2014; BARNES; ANDERSON; PHILLIPSON, 2012). Popularmente conhecida como açafrão da Índia (ORSOLIN; NEPOMUCENO, 2009; GRANDI, 2014), açafrão, açafroa, açafrão-da-terra, cúrcuma (GRANDI, 2014), gengibre dourado e mangarataia (ALMEIDA, 2006). Em outros países recebe outras nomeações, sendo, Yuquilla na região de Cuba, Turmeric na Inglaterra, Cúrcuma di Levante na Itália, Safran des Indes na França, Cúrcuma em Portugal, Kurkuma em Alemão, Haridra e Haldi pelos indianos (ALONSO, 1998; ALONSO, 2016).

Trata-se de uma planta herbácea, perene, de folhas grandes e longas e rizomas ovoides que podem alcançar até
$10 \mathrm{~cm}$ de comprimento. Quando cortados apresentam coloração amarelo avermelhado. Composta por flores amarela ou branca dispostas em espigas compridas. Exala cheiro forte, porém agradável e apresenta sabor picante e aromático (LORENZI; MATOS, 2008).

A Curcuma longa L. é uma planta originária da Índia e do sudeste da Ásia. Subsequentemente se difundiu pela América e em algumas regiões da Europa (ALONSO, 1998), na década de 80 foi introduzida no Brasil (ALMEIDA, 2006). Esta espécie se desenvolve em solo úmido e argiloso, portanto, se adapta bem na maioria dos países tropicais (ALONSO, 1998). É uma planta utilizada há 6.000 anos pela medicina Ayurveda, tradicional na Índia, sendo prescrita na prevenção e controle de desordens físicas que incluem: resfriado, comprometimento de vias aéreas, sinusite, infecções bacterianas, alterações hepáticas, diabetes, feridas, reumatismo, anorexia (WANG et al., 2014; ALMEIDA, 2006; ARAUJO; LEON, 2001). A parte da planta comumente utilizada são raízes/rizomas que são empregados como corante alimentício, tempero ou condimento alimentar (ALMEIDA, 2006; BARNES; ANDERSON; WANG et al., 2014).

\section{Constituintes e efeitos farmacológicos}

Os constituintes químicos da Curcuma longa L. incluem o óleo essencial, sendo rico em sesquiterpenos oxigenados, responsáveis pela característica aromática da planta (picante). Compostos de curcuminoides (curcumina, desmetoxicurcumina e bisdesmetoxicurcumina), responsáveis pela pigmentação dos rizomas (pigmento fenólico de cor amarelo avermelhado), tendo a curcumina como principal substância ativa (60 a 76\%), além de outros constituintes: carbinol, resina, amido, polissacarídeos (A, B, C e D), sais de potássio, açúcares, dentre outros constituintes (GRANDI, 2014; BASTOS; ROGERO; ARÊAS, 2009; ALMEIDA, 2006; ALONSO, 1998).

A curcumina é insolúvel em água, pouco solúvel em éter, e altamente solúvel em álcoois metílicos e etílicos (ORSOLIN; NEPOMUCENO, 2009). Apresenta alta estabilidade em diferentes $\mathrm{pH}$, principalmente em $\mathrm{pH}$ ácido do estômago (ALONSO, 2016).

Os principais componentes do óleo essencial desta planta são os sesquiterpenos ar-turmerone, $\alpha$-turmerone e $\beta$ -turmerone, seguido por $\alpha$-santaleno e ar-curcumeno (SINGH et al., 2010) ar-turmerol (SINGH; SINGH; MAURYA; 2002), curlone, zingiberene e curcumene (DOHARE et al., 2008).

\section{Aplicação Clínica}

A Curcuma longa L. apresenta diversas propriedades farmacológicas, dentre elas, destacam-se a sua ação antidiarreica, diurética, antiescorbútica, antiespasmódica, hepatoprotetora (GRANDI, 2014), anti-HIV, antiparasitário, inibidor da carcinogênese (ARAUJO; LEON, 2001), anti-inflamatória, antibacteriana, antiviral, antifúngica e antitumoral (BASTOS; ROGERO; ARÊAS, 2009), além de suas propriedades anticonvulsivantes (ALONSO, 2016), antiartrítico, redutor do nível de colesterol (ALMEIDA, 2006), sedativa e ação no sistema imune (YU; KONG; CHEN, 2002), antioxidante e neuroprotetora (KIM; KIM; YANG, 2014). 
A Curcuma longa L. apresenta potente ação anti-inflamatória (ARAÚJO; LEON, 2001). Este efeito é decorrente de diferentes mecanismos sobre a cascata do ácido araquidônico (cascata da inflamação) (ALONSO, 2016; BASTOS; ROGERO; ARÊAS, 2009; CHAINANI-WU, 2003). Neste contexto, a curcumina inibe diferentes moléculas envolvidas na inflamação, são elas: fosfolipase A, LOX - lipoxigenases, COX-2 - cicloxigenases, leucotrienos, tromboxanos, prostaglandinas, TNF- $\alpha$, MCP-1 (CHAINANI-WU, 2003), óxido nítrico, colagenase, elastase, hialuronidase (CHAINANI-WU, 2003; ROSA, 2009).

Há outras descrições sobre o mecanismo da ação anti-inflamatória da Curcuma longa L. (GÓNGORA et al., 2002; RAHMAN; BISWAS; KIRKHAM, 2006). Atua "sequestrando" espécies reativas de oxigênio em situações de estresse oxidativo celular, fato este que pode em parte interferir no processo inflamatório (GÓNGORA et al., 2002). A ação antibacteriana, antiviral, antifúngica e antitumoral da curcumina (RAHMAN; BISWAS; KIRKHAM, 2006), bem como o fato da mesma inibir a ativação do fator de transcrição AP-1, atuar em etapas que precedem a fosforilação do IKB- $\alpha$, bloquear NF- $\kappa$ B, podem estar relacionadas ao efeito anti-inflamatório da Curcuma longa L. (GÓNGORA et al., 2002).

A Curcuma longa L. vem também demostrando potente ação antioxidante devido à presença de seu composto curcuminoides. Segundo Manikandana et al (2009) e Alcalde; Del Pozo (2008) a curcumina atua na redução da peroxidação lipídica, além de aumentar a atividade de enzimas antioxidantes e a neutralização de radicais livres.

Os seus compostos curcuminoides e bisdemetoxicurcumina presentes no rizoma da Curcuma longa L, atua no controle e liberação da proteína ${ }_{\beta}$-amiloide. Esta proteína induz o estresse oxidativo e favorece a deterioração neural observada na doença de Alzheimer (PARK; KIM, 2002).

A Curcuma longa L. apresenta atividade em Sistema Nervoso Central (ALONSO, 2016). Dentre os efeitos em SNC encontra a ação antidepressiva. Estudos em ratos mostraram que os efeitos da Curcuma longa L. em dosagens entre 280 a $560 \mathrm{mg} / \mathrm{kg}$ expressaram ser mais efetivos do que o medicamento de referência (fluoxetina). Os autores sugerem que este efeito antidepressivo está relacionado com a atividade inibitoria da Monoamina Oxidase (MAO), sendo que a atividade da enzima MAO-A foi inibida com dose de $140 \mathrm{mg} / \mathrm{kg}$ e a MAO-B necessitou de uma dose de $560 \mathrm{mg} / \mathrm{kg}$, para ambas foram usadas dose-dependente. Desta forma, os autores concluíram que a Curcuma longa L. além de desempenhar papel sobre a depressão clínica pode ainda apresentar ação sobre desordens neurológicas e neurodegenerativas como a doença de Parkinson (YU; KONG; CHEN, 2002).

A ação neuroprotetora da Curcuma longa L. está relacionada aos compostos da curcumina. Estudos em ratos mostraram que a curcumina apresentou importante papel na prevenção de alterações cerebrais (efeito neuroprotetor). Neste estudo, preservou a integridade da membrana do cérebro de ratos contra efeitos adversos do álcool. Este efeito ocorreu através de sua ação antioxidante que é estabelecida pelo aumento dos níveis de glutationa e pela redução da peroxidação lipídica nas membranas neuronais (RAJAKRISHNAN et al., 1999). Os curcuminóides aplicados em ratas idosas inibiram o efeito deletério do envelhecimento sobre a disfunção mitocondrial, assim, minimizando e/ou bloqueando os distúrbios neurodegenerativos associados ao envelhecimento (RASTOGI et al., 2014).

A ação neuroprotetora da curcumina suprime o dano oxidativo, inibe peroxidases responsáveis por grande parte das citopatologias do Alzheimer (MUNIZ et al., 2012). A curcumina tem características hidrofóbicas que permite atravessar a barreira hematoencefálica e apresentar ação em tecido cerebral. Por meio desta descrição, juntamente a ação neuroprotetora a partir dos efeitos antiamiloidogênico, anti-inflamatório, antioxidante e quelante, a curcumina foi considerada bastante atraente como potencial terapêutica para doença de Alzheimer. Entretanto, apresenta baixa biodisponibilidade, pouca solubilidade em água e estabilidade em solução, além de rápida passagem pelo intestino e metabolização hepática, fatos que levam a necessidade de novas pesquisas, elaboração de métodos, a fim, de elucidar as lacunas do uso da curcumina em doenças neurodegenerativas (CHIN et al., 2013). Apresentação comercial atualmente disponível é o MOTORE (Achê) que contem extrato seco de Curcuma longa L. (MALLMANN, 2012).

A curcumina demonstrou ação anticonvulsionante em convulsões induzidas por pentilenotetrazol em camundongos com doses de $80 \mathrm{mg} / \mathrm{kg}$. Esta ação se deve ao fato da curcumina atuar sobre os receptores de adenosina A1 localizado na membrana da célula neuronal e não A2a (AKULA; KULKARNI, 2014). Este composto atua ainda promovendo melhoria da memória dependente, proteção de comprometimento cognitivo, prevenção e proteção contra a morte celular (KIM; KIM; YANG, 2014). Desta forma, verifica-se que a Curcuma longa L. tem mostrado efeitos benéficos ao Sistema Nervoso Central.

\section{Emprego clínico e posologia}

O uso da Curcuma longa L. tem sido crescente e pode ser administrada de diversas formas. Em geral pode ser por meio da decocção do rizoma á $1 \%$ de 2 a 3 vezes ao dia; Infusão de $20 \mathrm{~g} / 1$, sendo recomendado administrar 200 a $300 \mathrm{ml} / \mathrm{dia}$; Tintura de (1:10) recomendado tomar de 2,5 a $5 \mathrm{ml}$, de 1 a 3 vezes ao dia; Pó micronizado de $100 \mathrm{mg} /$ cápsula meia hora antes da primeira refeição; Extrato seco (5:1) recomenda-se 50 a $100 \mathrm{mg} /$ cápsula, ingerido de 2 a 3 vezes ao dia; Extrato padronizado a 95\% de curcuminoides, em cápsulas de $450 \mathrm{mg} /$ unidade 3 vezes ao dia e Extrato fluido (1:1), sendo recomendado de 30 a 80 gotas/dia divididas em 2 a 3 utilizações (ALONSO, 2016).

Para o tratamento da osteoartrite e artrite reumatoide ou uso destinado a ação anti-inflamatória e antioxidante a recomendação do extrato seco é de $500 \mathrm{mg}$ 2x ao dia (MALLMANN, 2012). Araujo (2015) descreve que para atividades; antitumoral, antioxidante, antiinflamatório, antimicrobiano a dose usual do extrato seco padronizado (95\%) varia de 300 a $600 \mathrm{mg} 3 \mathrm{x}$ ao dia. O uso na forma de infusão $(1,5 \mathrm{~g}$ de rizomas para $150 \mathrm{ml}$ de água) é recomendado numa dose de $150 \mathrm{ml} / 2 \mathrm{x}$ ao dia para ação antidispética e anti-inflamatória (BRASIL, 2011). Estudos em camundongos descreveram que a Curcuma longa L. apresentou ação anticonvulsionante com doses de 80 mg/kg (AKULA; KULKARNI, 2014). Em ratos apresentou ação antidepressiva com dosagens entre 280 a $560 \mathrm{mg} / \mathrm{kg}$ (YU; KONG; CHEN, 2002). 


\section{Efeitos adversos e contra-indicações}

A Curcuma longa L. apresenta ação fotossensibilizante para pacientes que recebem o tratamento diário. Assim, o indivíduo fica susceptível à alterações cutâneas quando expostos ao sol (radiação ultravioleta). Neste caso, é recomendável o uso de protetor solar, óculos de sol e chapéu, bem como o hábito de evitar a exposição solar (ALONSO, 2016).

Para indivíduos que apresentam estômago hipersensível, o uso da Curcuma longa L. pode agravar os sintomas (FINTELMANN; WEISS, 2014; GRANDI, 2014). Já o uso prolongado ou em altas doses, pode desencadear úlceras gástricas. Desta forma, seu uso fica restrito para pacientes que apresentam úlceras gástricas e uso cauteloso para aqueles que apresentam histórico pessoal ou familiar de úlceras (FETROW; AVILA, 2000; ALONSO, 2016). Ainda é contra indicado para portadores de distúrbios hemorrágicos e obstrução de ductos biliares (FETROW; AVILA, 2000; ALONSO, 2016; GRANDI, 2014).

A Curcuma longa L. é descrita como estimulante hormonal, assim, pode induzir ao aborto (FETROW; AVILA, 2000). O óleo essencial em doses inadequadas também foi descrito como abortivo (ALONSO, 2016). Portanto, o uso da cúrcuma para gestantes, lactantes e crianças é contra indicado (ALONSO, 2016; GRANDI, 2014).

Esta planta foi também descrita como sendo neurotóxica quando administrada em doses inadequadas (ALONSO, 2016). Pode induzir dermatite de contato alérgica (FETROW; AVILA, 2000; ALONSO, 2016). Não deve ser indicada para indivíduos que apresentem sensibilidade ou alergia a curcumina ou a qualquer outro componente da planta (GRANDI, 2014).

Outras contra indicações incluem pacientes que estejam fazendo uso de medicações que alterem suas características de coagulação como antiagregantes plaquetários, anticoagulantes, heparina de baixo peso molecular e agentes trombolíticos (MALLMANN, 2012).

\section{Interação farmacocinética}

A Curcuma longa L. aumenta os níveis sanguíneos de diversos medicamentos (GRANDI, 2014). Portanto, seu uso deve ser realizado sob orientação profissional, a fim, de evitar os efeitos de interações medicamentosas (ver tabela 1).

A Curcuma longa L. apresenta princípios ativos com atividade anticoagulante, portanto, o uso concominante à agentes anticoagulantes pode desencadear efeitos aditivos sobre as plaquetas, aumentando assim, o risco à hemorragias (ALONSO, 2016; FETROW; AVILA, 2000; GRANDI, 2014). O uso de anti-inflamatórios não-esteroides pode também apresentar inibição da função plaquetária, desencadeando susceptibilidade a sangramentos (FETROW; AVILA, 2000).

Foram observados que a Curcuma longa L. apresenta interações induzindo o metabolismo de agentes imunossupressores, resultando na aceleração do metabolismo, aumentando sua excreção, assim, reduzindo os efeitos destes medicamentos com consequente interferência no tratamento (FETROW; AVILA, 2000).

A curcumina juntamente ao paclitaxel (Taxol®) inibiu as metástases pulmonares de forma significativa quando comparados com a curcumina e o paclitaxel (Taxol®) isoladamente. Os autores descrevem que este resultado se deva a inibição significativa do NF-KB (AGGARWAL et al., 2005).

Autores descrevem que a Curcuma longa L. apresenta ação sobre o câncer de mama. Grandi (2014) descreve que a genisteína usada simultaneamente a curcumina promoveu diminuição do crescimento de células cancerosas de mama. Este efeito de diminuição do crescimento de células de câncer de mama foi também descrito a partir da curcumina, sendo que esta reduziu a atividade citotóxica da camptotecina (SOMASUNDARAM et al., 2002).

A curcumina e a demetoxicurcumina demonstraram inibição das funções intestinais da glicoproteína P. (AMPASAVATE et al., 2010). A associação da curcumina com ciclosporina, pode ser usado como coadjuvante na quimioterapia, isto porque apresenta ação bloqueadora da resistência à proliferação de células T. (GRANDI, 2014). Segundo Alonso (2016), pode ainda interferir na absorção e eficácia da irinotecán e intensificar a atividade dos fármacos Oseltamivir e Cotrimoxazol.

Tabela 1: Interação medicamentosa entre a Curcuma longa L. e outras drogas.

\begin{tabular}{l|l|l}
\hline \multicolumn{1}{c|}{ Droga } & \multicolumn{1}{|c}{ Efeito da Interação (Curcuma longa L. X Drogas) } & \multicolumn{1}{c}{ Referência } \\
\hline Anticoagulante & $\begin{array}{l}\text { Desencadea efeitos aditivos sobre as plaquetas. } \\
\text { Aumenta o risco a hemorragias. }\end{array}$ & $\begin{array}{l}\text { (ALONSO, 2016; FETROW; } \\
\text { AVILA, 2000; GRANDI, 2014) }\end{array}$ \\
\hline $\begin{array}{l}\text { Anti-inflamatórios não } \\
\text { esferoidais (AINE) }\end{array}$ & $\begin{array}{l}\text { Inibe a função plaquetária, gera susceptibilidade a sangra- } \\
\text { mentos. }\end{array}$ & (FETROW; AVILA, 2000) \\
\hline Imunossupressores & $\begin{array}{l}\text { Acelera o metabolismo, aumenta sua excreção. Reduz os } \\
\text { efeitos do medicamento. }\end{array}$ & (FETROW; AVILA, 2000) \\
\hline Paclitaxel (Taxol®) & Potencialização dos efeitos do medicamento. & (AGGARWAL et al., 2005) \\
\hline Irinotecán & Interfere na absorção e eficácia do medicamento. & (ALONSO, 2016) \\
\hline Oseltamivir & Intensifica a atividade do fármaco. & (ALONSO, 2016) \\
\hline Cotrimoxazol & Intensifica a atividade do fármaco. & (ALONSO, 2016) \\
\hline
\end{tabular}

\section{Considerações Finais}

A pesquisa permitiu verificar a importância da Curcuma longa L. e seu grande potencial de utilização. A Curcu- ma longa L. tem apresentado atividades farmacológicas para diferentes sistemas e consequentemente vem sendo aplicada para diversas disfunções. Os principais efeitos descritos são anti-inflamatório e provável efeito neuroprotetor com poten- 
cial para o desenvolvimento de medicamentos. Os principais componentes ativos da Curcuma longa L. que apresentam ação em sistema nervoso central e anti-inflamátório são os curcuminóides, sendo descritas principalmente ações antioxidantes.

Diante disso, para a avaliação do potencial enquanto planta medicinal faz-se necessários estudos de validação farmacológica para o emprego clínico objetivando benefícios à saúde humana.

\section{Referências}

ABRANCHES, M. V. Plantas Medicinais e Fitoterápicos - Abordagem teórica com enfâse em nutrição. Viçosa: A.S. Sistemas, 2015.

AGGARWAL, B. B.; et al. Curcumin Suppresses the Paclitaxel-Induced Nuclear Factor-KB Pathway in Breast Cancer Cells and Inhibits LungMetastasis ofHumanBreast Cancer inNudeMice. Clin Cancer Res. v. 11, n. 20, out. p. $7490-7498,2005$.

AKULA, K. K.; KULKARNI, S. K. Effect of Curcumin Against PentylenetetrazolInduced Seizure Threshold in Mice: Possible Involvement of Adenosine A1 Receptors. Phytother. Res. v. 28, p. 714-721. 2014.

ALCALDE, M. T.; DEL POZO, A. Nuevos despigmentantes cutâneos (XII). Tetrahidrocurcuminóides. OFFARM. v.27, n. 5, p. 130-131, 2008.

ALMEIDA, L. P. Caracterização de pigmentos da Curcuma longa, L., avaliação da atividade antimicrobiana, morfogênese in vitro na produção de curcuminóides e óleos essenciais. Dissertação de Doutorado. Faculdade de Farmácia da Universidade Federal de Minas Gerais. Belo Horizonte, 2006, p. 120.

ALONSO, J. Curcuma. In: ALONSO, J. Tratado de Fitofarmácos e Nutracêuticos. São Paulo: A C Farmacêutica, 2016. p. 364 - 373.

ALONSO, J. R. Tratado de fitomedicina: bases clinicas y farmacológicas. Argentina: Isis, 1998. p. 439 - 444.

AMPASAVATE, C. et al. Effects of Curcuma spp. on P-glycoprotein function. Phytomedicine. v. 17, p. 506-512, 2010 .

ARAUJO, A. Curcuma longa - antitumoral, antioxidante, antiinflamatório, antimicrobiano. Pharma Nostra. 2015.

ARAUJO, C. A. C.; LEON, L. L. Biological activities of Curcuma longa L. Mem. Inst. Oswaldo Cruz, Rio de Janeiro. v. 96. n. 5, p. 723-728, 2001.

BARNES, J.; ANDERSON, L. A.; PHILlIPSON, J. D. Fitoterápicos. 3. ed. Porto Alegre: Artmed, 2012. p. 720.

BASTOS, D. H. M.; ROGERO, M. M.; ARÊAS, J. A. G. Mecanismos de ação de compostos bioativos dos alimentos no contexto de processos inflamatórios relacionados à obesidade. Arq Bras Endocrinol Metab. v. 53, n. 5, p. 646-656, 2009.

BRASIL. Agência Nacional de Vigilância Sanitária. Formulário de Fitoterápicos da Farmacopéia Brasileira. Agência Nacional de Vigilância Sanitária. Brasília: Anvisa, 2011. p. 126.

CARVAlHOS, J. C. T. Formulário Médico-Farmacêutico de Fitoterapia. 3. ed. São Paulo: Pharmabooks, 2012. p. 365 .

CHAINANI-WU. N. Safety and Anti-Inflammatory Activity of Curcumin: A Component of Tumeric (Curcuma longa). THE JOURNAL OF ALTERNATIVE AND COMPLEMENTARY MEDICINE. v. 9, n. 1, p. 161-168, 2003.

CHIN, D. et al. Neuroprotective Properties of Curcumin Merits and Limitations. Current Medicinal Chemistry • v. 20, n. 32. Ago. 2013.

DOHARE, P. et al. Neuroprotective efficacy and therapeutic window of curcuma oil: in rat embolic stroke model.

BioMed Central. v. 8, n. 55, 2008. p. 1-20.

ELDIN, S.; DUNFORD, A. Fitoterapia na atenção primária à saúde. Barueri: Manole, 2001. p. 147.

FETROW, C. W.; AVILA, J. R. Manual de Medicina Alternativa para o Profissional. Rio de Janeiro: Guanabara Koogan, 2000. p. 245 - 248.

FINTELMANN, V.; WEISS, R. F. Manual de Fitoterapia. Rio de Janeiro: Guanabara Koogan, 2014. p. 132 - 135.

FIRMO, W. C. A. et al. Contexto histórico, uso popular e concepção científica sobre plantas medicinais. Cad. Pesq., São Luís, v. 18, n. especial, dez. 2011, p. 90 - 95.

GIRALDI, M.; HANAZAKI, N. Uso e conhecimento tradicional de plantas medicinais no Sertão do Ribeirão, Florianópolis, SC, Brasil. Acta bot. bras. v. 24, n. 2, p. 395-406. 2010.

GÓNGORA, L. et al. Effects of caffeoyl conjugates of isoprenyl-hydroquinone glucoside and quinic acido $n$ leukocyte function. Life Sci. v. 71, n. 25, p. 2995 - 3004, 2002.

GRANDI, T. S. M. Tratado das plantas medicinais: Mineiras, Nativas e Cultivadas. Belo Horizonte: Adaequatio Estúdio, 2014. p. 1076-1077.

KIM, M. H.; KIM, S. H.; YANG, W. M. Mechanisms of action of phytochemicals from medicinal herbs in the treatment of Alzheimer's disease. Planta Med, v. 80, n. 15, p. 1249-58, Oct 2014.

LORENZI, H.; MATOS, A. Plantas medicinais no Brasil: 
nativas e exóticas. 2. Ed. Nova Odessa: Instituto Plantarum, 2008. p. 541-542.

MANIKANDANA, R. et al. Anti-cataractogenic effect of curcumin and aminoguanidine against selenium-induced oxidative stress in the eye lens of Wistar rat pups: An in vitro study using isolated lens. Chimico-Biological Interactions, v. 181, p. 2002-2009, 2009.

MALLMANN, G. Guarulhos: Aché Laboratórios Farmacêuticos S.A., 2012.

MUNIZ, R. M. C. C. et al. Plantas Medicinais da RENISUS de Atuação Central. Infarma, v. 24, n. 1-3, p. 75- 80, 2012.

ORSOLIN, P. C.; NEPOMUCENO, J. C. Potencial carcinogênico do açafrão (Curcuma longa L.) identificado por meio de teste para detecção de clones de tumor em Drosophila melanigaster. Revista do Núcleo Interdisciplinar de Pesquisa e Extensão do UNIPAM. Patos de Minas: UNIPAM, v. 6, p. 55-69. Out, 2009.

PARK, S.; KIM, D. Discovery of Natural Products from Curcuma longa that Protect Cells from Beta-Amyloid Insult: A Drug Discovery Effort against Alzheimer's Disease. Journal Of Natural Products. v. 65, n. 9, p. 1227 1231, 2002.

RAHMAN, I.; BISWAS, S. K.; KIRKHAM, P. A. Regulation of inflammation and redox signaling by dietary polyphenols. Biochem Pharmacol. v. 72, n. 11, p. $1439-$ 1452, 2006.

RAJAKRISHNAN, V. et al. Neuroprotective Role of Curcumin from Curcuma longa on Ethanol-induced Brain Damage. Phytother. Res. v.13, p. 571-574. 1999.

RASTOGI, M. et al. Protective effect of curcuminoids on age-related mitochondrial impairment in female Wistar rat brain. Biogerontology. v. 15, p. 21-31. 2014.

ROSA, C. de O. B. Avaliação do efeito de compostos naturais - curcumina e hesperidina - na hiperlipidemia induzida em coelhos. 2009. 98 f. Tese (Doutorado em Bioquímica Agrícola) - Universidade Federal de Viçosa, Vicosa, 2009.

ROSSATO, A. E. et al. Fitoterapia racional: aspectos taxonômicos, agroecológicos, etnobotânicos e terapêuticos. Florianópolis: DIOESC, 2012. p. 211.

SOMASUNDARAM, R. et al. Inhibition of Cytolytic T Lymphocyte Proliferation by Autologous CD4/CD25 Regulatory T Cells in a Colorectal Carcinoma Patient Is Mediated by Transforming Growth Factor- $\beta$. Câncer Research, v. 62, p. 5267-5272, Set. 2002.

SINGH, G.; SINGH, O.P.; MAURYA, S. Chemical and biocidal investigations on essential oils of some Indian Curcuma species. Progress in Crystal Growth and

Characterization of Materials. v.45, p.75-81, 2002.
SINGH, G. et al. Comparative study of chemical composition and antioxidant activity of fresh and dry rhizomes of turmeric (Curcuma longa Linn.). Food and Chemical Toxicology. v. 48. n. 4. 2010. p. 1026-1031.

TORQUATTO, J. Fitoterapia: vantagens, risco e ação dos fitoterápicos. São Paulo, 2013. p. 121.

WANG, X. et al. Effects of curcuminoids identified in rhizomes of Curcuma longa on BACE-1 inhibitory and behavioral activity and lifespan of Alzheimer's disease Drosophila models. BMC Complement Altern Med, v. 14, p. 88, 2014.

YU, Z. F.; KONG, L. D.; CHEN, Y. Antidepressant activity of aqueous extracts of Curcuma longa in mice. J Ethnopharmacol, v. 83, n. 1-2, p. 161-5, Nov 2002.

Recebido em: 18/04/2016 Aceito em: 20/11/2016 